\title{
Reduced Recombination and Capacitor-like Charge Buildup in an Organic Heterojunction
}

\author{
Authors: Kyra N. Schwarz ${ }^{1,3}$, Paul B. Geraghty",3, Valerie D. Mitchell ${ }^{2,3}$, Saeed-Uz-Zaman \\ Khan $^{4}$, Oskar J. Sandberg ${ }^{6}$, Nasim Zarrabi ${ }^{6}$, Bryan Kudisch ${ }^{1}$, Jegadesan Subbiah ${ }^{2,3}$, Trevor A. \\ Smith $^{3}$, Barry P. Rand ${ }^{4,5}$, Ardalan Armin ${ }^{6}$, Gregory D. Scholes ${ }^{1,3 *}$, David J. Jones ${ }^{2,3}$, Kenneth P. \\ Ghiggino $^{3 *}$
}

Affiliations:

${ }^{1}$ Department of Chemistry, Princeton University, Princeton, NJ 08544, USA

${ }^{2}$ Bio21 Institute and ${ }^{3}$ School of Chemistry, University of Melbourne, Parkville, VIC 3010, Australia

${ }^{4}$ Department of Electrical Engineering and ${ }^{5}$ Andlinger Center for Energy and the Environment, Princeton University, Princeton, NJ 08544, USA

${ }^{6}$ Department of Physics, Swansea University, Singleton Park, Swansea, SA2 8PP, Wales, United Kingdom

ABSTRACT: Organic photovoltaic (OPV) efficiencies continue to rise, raising their prospects for solar energy conversion. However, researchers have long considered how to suppress the loss of free carriers by recombination-poor diffusion and significant Coulombic attraction can cause electrons and holes to encounter each other at interfaces close to where they were photogenerated.

20 Using femtosecond transient spectroscopies, we report the nanosecond grow-in of a large transient Stark effect, caused by nanoscale electric fields of $\sim 487 \mathrm{kV} / \mathrm{cm}$ between photogenerated free carriers in the device active layer. We find that particular morphologies of the active layer lead to an energetic cascade for charge carriers, suppressing pathways to recombination, which is $\sim 2000$ times less than predicted by Langevin theory. This in turn leads to the build-up of electric charge in donor and acceptor domains - away from the interface-resistant to bimolecular recombination. Interestingly, this signal is only experimentally obvious in thick films, due to the different scaling of electro-absorption and photo-induced absorption signals in transient absorption spectroscopy. 
Rather than inhibiting device performance, we show that devices up to $600 \mathrm{~nm}$ thick maintain efficiencies of $>8 \%$ because domains can afford much higher carrier densities. These observations suggest that with particular nanoscale morphologies, the bulk heterojunction can go beyond its established role in charge photogeneration, and can act as a capacitor, where adjacent free charges are held away from the interface and can be protected from bimolecular recombination.

\section{INTRODUCTION:}

One of the greatest challenges for light harvesting technologies is the successful collection and usage of photogenerated charged species. The majority of organic photovoltaic (OPV) devices use a bulk heterojunction for their active layer-consisting of donor and acceptor domains-where molecular structural complexity is viewed as a challenge to be overcome for harvesting free carriers $^{1,2}$. In contrast, photosynthetic light-harvesting complexes actually rely on nanoscale intricacies to create cascades that separate and hold mutually-attracted charges at a distance for optimal quantum efficiency ${ }^{3}$.

15 The organic bulk heterojunction's nanoscale morphology is intimately involved in each stage of the energy conversion process. Following light absorption, excitons diffuse to a donor-acceptor interface where charges can be generated, that in turn are transported to their respective electrodes in order to generate photocurrent and photovoltage ${ }^{4}$. In current high-performing OPVs, charge generation is fairly efficient and the recombination of separated charges can be considered the largest limitation of efficiency ${ }^{5,6}$. This is particularly important for scaling up organic solar cells to large areas using high throughput fabrication systems. ${ }^{7}$ Large scale deposition demands junctions thicker than conventional active layers i.e. larger than $100 \mathrm{~nm}$. Due to low mobilities and significant bulk carrier recombination, organic solar cells often lose their efficiency in the thick junction limit. Only a few systems so far have shown promise in this regard - delivering high 25 efficiencies even when the junction thickness exceeds $500 \mathrm{~nm} \cdot{ }^{8-10}$ It has been demonstrated that the reason behind this is reduced recombination with respect to the Langevin encounter limit., ${ }^{9,11}$ Amongst the few systems exhibiting reduced bimolecular recombination, the highest reduction factor (relative to the Langevin rate) so far has been 800 times. $^{8}$

To understand the fate of photogenerated charges, we track their time-resolved photoinduced absorption and we also examine the influence of local electric fields formed by charge carriers, 
that can be detected by the use of an electro-absorption or Stark signal ${ }^{12}$. This effect occurs when an electric field perturbs an optical transition, which is sensitive to either its change in dipole or change in polarizability in the presence of the field. This signature can be observed when a macroscopic electric field is applied to a light absorbing species, as in Stark spectroscopy ${ }^{12-15}$. It can also be caused directly by photo-generated charges, where it has been used as a probe of charge separation at the nanoscale ${ }^{16-18}$, including for sub-picosecond charge transfer in bulk heterojunctions ${ }^{19-22}$.

In this work, we demonstrate that the bulk heterojunction morphology can play an unforeseen role-keeping adjacent free carriers apart via capacitive charging. An active layer morphology consisting of pure and intermixed domains is able to block a key recombination pathway to triplet excitons and suppress bimolecular recombination up to 2000 times greater than predicted by the Langevin model. This is the largest reduction factor so far reported in a technologically relevant organic solar cell. This occurs concurrently with large electric fields that build-up following charge generation in these systems, and are maintained across domains for nanoseconds, resembling the charging of a capacitor. To our knowledge, this interdomain capacitive effect, manifested by a local electric field buildup with a magnitude of $\sim 487 \mathrm{kV} / \mathrm{cm}$, is unprecedented in any photovoltaic system. The dielectric constant of the material dictates that the dielectric polarization of intervening amorphous and interfacial domains in the presence of such fields must further protect charges, much like the dielectric layer in a macroscopic capacitor. Rather than being detrimental, we show that this electric field buildup is related to the prevention of non-radiative loss pathways and substantial improvements in device performance, including in thick junction devices.

\section{EXPERIMENTAL SECTION}

\section{Sample and Device Preparation}

BQR was prepared according to a synthetic procedure published previously ${ }^{23}$. To prepare neat films, BQR and PC71BM (Nano-c, 99\%) were first dissolved in chloroform (HPLC-grade) to a concentration of $10 \mathrm{mg} / \mathrm{mL}$. To prepare blend films, solutions of BQR and PC $71 \mathrm{BM}$ were prepared by dissolving respectively in chloroform to a concentration of $13.3 \mathrm{mg} / \mathrm{mL}$ (95 nm 'thin' films) $16.5 \mathrm{mg} / \mathrm{mL}$ (110 nm films), $20 \mathrm{mg} / \mathrm{mL}$ (140 nm ‘thick’ films) and 32mg/mL (220 nm films) and then combining in equal parts for 1:1 blends w/w, and a ratio of 95:5 for majority BQR active layers. The substrates were cleaned by sonicating sequentially in $1 \mathrm{M} \mathrm{NaOH}$, distilled water, 
acetone, isopropanol, and dichloromethane, followed by 15 min of UV/ozone treatment. All films were cast onto clean glass substrates via spin coating at 1000 rpm (2000 acceleration) for 30 seconds. Solvent vapor annealing was conducted in a tetrahydrofuran (THF) atmosphere with an exposure time of 20 seconds.

Organic photovoltaic devices were processed on pre- patterned indium tin oxide (ITO) coated glass substrates with a sheet resistance of $15 \Omega /$ sq. The conventional devices were fabricated with a device geometry of glass/ITO/PEDOT:PSS/active layer/Ca/Al. The ITO-coated glass substrates were cleaned by ultrasonic treatment in acetone and isopropyl alcohol for 15 minutes each and subsequently treated in UV-Ozone for 15 minutes. A thin layer (30 nm) of PEDOT:PSS (Clevios P VP AI 4083, filtered at $0.45 \mu \mathrm{m}$ ) was spin-coated onto the ITO surface. After baking at $150{ }^{\circ} \mathrm{C}$ for 10 minutes, the substrates were transferred into a nitrogen-filled glove box. Subsequently, the active layer was spin-coated from blend chloroform solutions with weight ratio of donor and $\mathrm{PC}_{71} \mathrm{BM}$ at 1:1 Then, the substrates were placed in a glass Petri dish containing $1 \mathrm{~mL}$ THF for solvent vapor annealing (SVA). After SVA treatment, the films were transferred to a metal evaporation chamber and a bilayer cathode consisted of Ca (30 nm) capped with $\mathrm{Al}(100 \mathrm{~nm})$ was deposited through a shadow mask (active area was $0.1 \mathrm{~cm}^{2}$ ) at approximately $1 \times 10^{-6}$ Torr.

\section{Steady-state Spectroscopy}

Absorption spectra of all films were recorded using a Varian Cary 50 UV-Vis spectrophotometer. Fluorescence spectra were recorded on a Varian Eclipse spectrofluorimeter using an excitation wavelength of $600 \mathrm{~nm}$, where all spectra were corrected. The optical constants were obtained through spectroscopic ellipsometry using a J.A Woollam Co. M-2000 ellipsometer.

\section{Electrical Charge Transport and Recombination Measurements}

RPV measurements were conducted using a Pharos PH1-10 laser at the second harmonic wavelength of $514 \mathrm{~nm}$. The pulse duration was approximately $290 \mathrm{fs}$ and the fluence was lowered to less than a $\mathrm{nJ} / \mathrm{cm}^{2}$ using neutral density filters. The repetition rate of the experiment was $20 \mathrm{~Hz}$. The device was mounted in a closed metal box acting as a Faraday cage to minimise the pick-up noise. The transients were recorded using a Rohde\&Schwartz RTM3004 oscilloscope. A homemade decade variable resistance box was used to vary the series resistance of the circuit from 50 Ohms to 1 MOhms. The transient double injection measurements were done using the same oscilloscope and a Keysight 33500B function generator was used to apply the voltage pulse to the 
devices in forward bias. Steady-state currents were recorded using a Keithley 2450 source-meter unit.

\section{Electro-optical Device Simulations}

For the device simulations, a numerical steady-state drift-diffusion model was used. ${ }^{24}$ The device

model solves the drift-diffusion current equations in conjunction with the charge carrier continuity equations and the Poisson equation for a given applied voltage and charge carrier generation rate at AM1.5G incident light intensity. The recombination rate between charge carriers was taken to be bimolecular, with a recombination coefficient reduced by a factor of $\gamma$ compared to Langevin recombination. Furthermore, the carrier densities at the contacts are assumed to be in thermal equilibrium. The parameters used are listed in the supporting information. The generation rate was calculated based on the optical constants of the device stack using a transfer matrix model that takes into account the back-electrode reflection and interference effects of the optical electric field inside the device. ${ }^{25}$

\section{Transient Absorption Spectroscopy}

Transient absorption spectroscopy was undertaken on a $92 \mathrm{kHz}$ repetition-rate laser and reproduced on a $1 \mathrm{kHz}$ system, both of which are described here.

For the high repetition rate system, a mode-locked Ti:sapphire oscillator (Coherent, Mira Seed) seeded a Ti:sapphire regenerative amplifier system (Coherent, RegA 9050) to produce pulses of $\sim 50$ fs duration at a repetition rate of $92 \mathrm{kHz}$ and a wavelength centered at $800 \mathrm{~nm}$. The $600 \mathrm{~nm}$ and $553 \mathrm{~nm}$ pump beams were generated with an optical parametric amplifier (OPA9450, Coherent). The pump beam was mechanically chopped at $\sim 3.5 \mathrm{kHz}$, and the arrival time of the pump pulses relative to the probe was manipulated using a variable optical delay line (Newport, UTS150PP with ESP 300 controller). The broadband probe was derived from the residual $800 \mathrm{~nm}$ beam focused onto a $3 \mathrm{~mm}$ sapphire substrate (Crystal Systems) for measurements in the visible region (450-800 nm) and a 5 mm undoped YAG substrate (Crystal Systems) for the infrared region (800-1400 nm). After passing through the sample, the probe beam was analyzed with a CMOS detector (Ultrafast Systems) at 7077 spectra/s, and the excess $800 \mathrm{~nm}$ laser fundamental was removed using low-and high-pass filters for the visible and IR regions respectively. For all measurements unless otherwise noted, the pump beam was attenuated to a pulse power of 3.2 
$\mathrm{nJ} /$ pulse with a spot area of $1.3 \times 10^{-3} \mathrm{~cm}^{2}$, giving a pump power density of $2.5 \mu \mathrm{J} / \mathrm{cm}^{2}$. Nitrogen was blown over films for all measurements. For further details on this experimental setup, see ref 26 .

Transient absorption experiments with a time delay of $>10$ ns were carried out using Helios-EOS, (Ultrafast Systems) where the pump beam was used as described above. Probe pulses ( 2 ns duration) were generated by built-in photonic crystal fiber-based supercontinuum pulsed light source with an electronic delay between pump and probe.

Ultrafast TA measurements were additionally conducted and replicated with a $1 \mathrm{kHz}$ Ti:sapphire regenerative amplifier system (Coherent Libra) with an output of $800 \mathrm{~nm}$ pulses with $\sim 45 \mathrm{fs}$ pulsewidth and approximately $4 \mathrm{~W}$ power. The pump arm of the Libra output was directed to an optical parametric amplifier (Light Conversion OPerA), and thereby converted into $600 \mathrm{~nm}$ pulses. The probe arm of the Libra output was directed to a commercial transient absorption spectrometer (Ultrafast System, Helios) and was used in the generation of a visible and NIR continua, from 450-800 nm and 800-1500 nm respectively. Optical filters were incorporated in order to separate out excess $800 \mathrm{~nm}$ remaining from the continua generation. Referencing of the probe spectra using a second camera was required to achieve adequate signal-to-noise at low pump fluence. The pump beam spot size was measured by analyzing the image obtained by a digital CCD camera (Thorlabs Inc.) with Thorcam Software (Thorlabs Inc.) placed at the pump probe overlap. The size of the pump beam at ca. $600 \mathrm{~nm}$ was approximately $3.8 \times 10^{-3} \mathrm{~cm}^{2}$. The pump

20 power was measured with a high-sensitivity optical power sensor (Coherent, Santa Clara, California). Incident pump power in the visible was $\sim 9.5 \mathrm{~nJ}$ per pulse, giving a pump fluence of $2.5 \mu \mathrm{J} / \mathrm{cm}^{2}$.

For both measurements, unless otherwise indicated, an excitation density of $2.5 \mu \mathrm{J} / \mathrm{cm}^{2}$ was used, to prevent artifacts associated with exciton-exciton ${ }^{27}$ and exciton-charge annihilation ${ }^{28,29}$. The relative orientation of pump and probe polarization was $54.7^{\circ}$ (magic angle) and all spectra were corrected for the chirp of the supercontinuum probe.

\section{Grazing Incidence Wide Angle X-ray Spectroscopy (GIWAXS)}

GIWAXS measurements were conducted at the SAXS/WAXS beamline of the Australian synchrotron. The substrates were silicon wafers that had been sonicated in acetone and isopropanol for 30 min each followed by 15 min of UV/ozone treatment. The measurements were performed 
with an X-ray energy of $11 \mathrm{keV}$ and a range of incident angles from $\Omega=0.025-0.5$ in $0.01-0.05$ increments to allow signal optimization near the critical angle of the polymer film but below the critical angle of the substrate. Data from GIWAXS experiments were analyzed using a customized version of NIKA 2D based in IgorPro.

\section{Steady-state Electro-absorption Measurements}

Electro-absorption (EA) samples were fabricated on pre-patterned indium tin oxide (ITO) substrates by spin coating $\mathrm{BQR}: \mathrm{PC}_{71} \mathrm{BM}(1: 1)$ solution. $10 \mathrm{mg} / \mathrm{mL}$ stock solution was made in chloroform. Spin coating was conducted in $\mathrm{N}_{2}$ glovebox at 1000 r.p.m. for $30 \mathrm{~s}$ and annealed at $100^{\circ} \mathrm{C}$ for $10 \mathrm{~min}$. Later, $10 \mathrm{~nm}$ of bathocuproine (BCP) was vacuum evaporated on the BQR:PC ${ }_{71} \mathrm{BM}$ film, followed by $100 \mathrm{~nm}$ of aluminum (Al). Profilometer measurements of the films gave a thickness of $73.6 \mathrm{~nm}$ for the active layer, giving a total of $83.6 \mathrm{~nm}$ thickness between electrodes.

EA measurements were conducted in reflection mode, with light beam reflecting off the back $\mathrm{Al}$ contact of the EA sample, passing the active layer twice. The direct reflected light was collected using a Si photodetector. The sample was biased with $-1 \mathrm{~V} \mathrm{DC}$, to prevent charge injection. $1 \mathrm{kHz}$ AC modulating voltage of amplitudes $0 \mathrm{~V}-5 \mathrm{~V}$, at an interval of $200 \mathrm{mV}$ was applied, superimposed on the DC voltage. The difference in reflection from the sample with applied AC voltage was measured using a lock-in amplifier, detecting the second harmonic signal, $2 \omega$, from the photodetector.

\section{Computational Methods}

Density functional theory (DFT) calculations were performed using Gaussian $16 .{ }^{30}$ Geometry optimization and calculation of the dipole moment and polarizability was performed using the 631G(d,p) basis set and the B3LYP functional.

\section{RESULTS AND DISCUSSION:}


We report studies of thin films of the electron donor BQR (benzodithiophene-quaterthiophenerhodanine $)^{23}$ (Fig. 1a), and blends with an electron acceptor $\mathrm{PC}_{71} \mathrm{BM}\left([6,6]\right.$-phenyl-C ${ }_{71}$ butyric acid methyl ester). Neat BQR films consist of both amorphous and aggregate species, where $\pi$ stacked molecules can be identified by their vibronic structure on the low-energy end of the absorption spectrum (580-700 nm) (Fig 1b). This aggregation is somewhat interrupted when incorporated into a film with $\mathrm{PC}_{71} \mathrm{BM}$ (Fig. 1b lower).

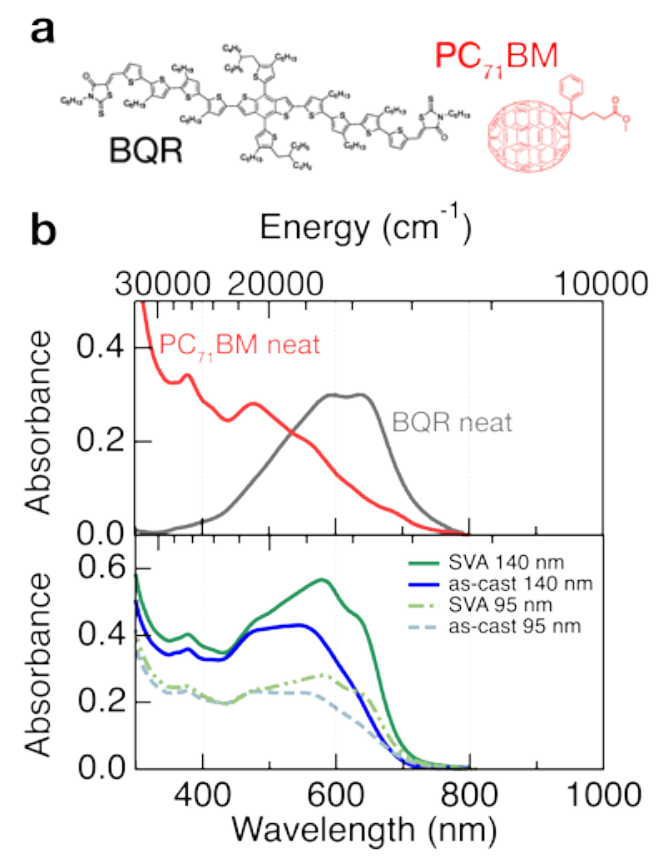

Figure 1. (a) The chemical structures of $B Q R$ and $P_{71} B M$. (b) Absorbance of neat donor $\mathrm{BQR}$ and neat acceptor $\mathrm{PC}_{71} \mathrm{BM}$ films (upper), and of 1:1 BQR:PC ${ }_{71} \mathrm{BM}$ blend films (lower), with a $\sim 140 \mathrm{~nm}$ thick active layer; as-cast (blue solid) and SVA (green solid). Thinner blend films with a $\sim 95 \mathrm{~nm}$ thick active layer are shown in dashed lines: as-cast (blue dashed) and SVA (green dashed). 
a

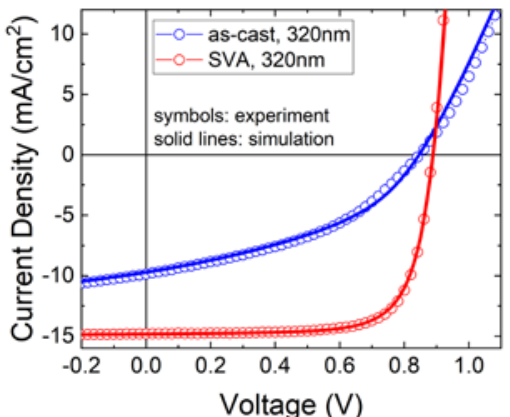

C

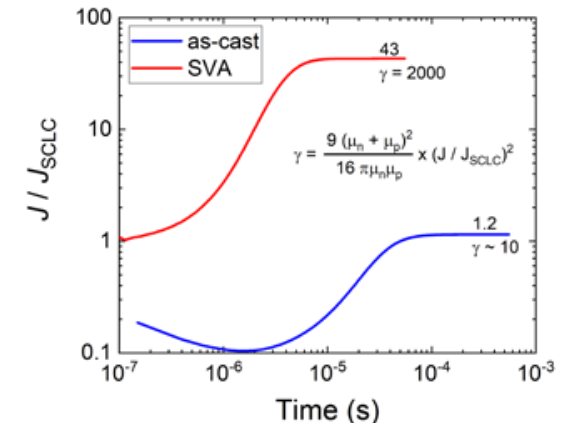

b

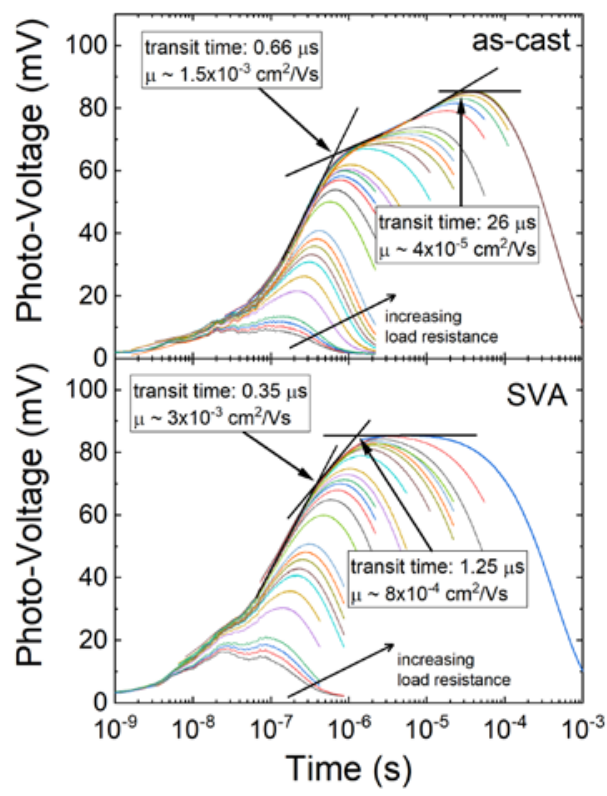

Figure 2. (a) The experimental $J-V$ curves of $320 \mathrm{~nm}$ thick as-cast (blue circles), with PCE $=3.45 \%$ and $\mathrm{FF}=41.4 \%$, and SVA device (red circles), with PCE $=9.75 \%$ and FF $=74.4 \%$. The simulated $J$ - $V$ curves, using the electro-optical device model, are indicated by the corresponding solid lines. (b) Resistance-dependent Photo-Voltage transients for as-cast (upper) and (SVA) (lower) device. The extracted transit times with the associated mobilities $(\mu)$ are indicated. (c) Double injection current transient measurements on as-cast (blue) and SVA device (red) for the determination of the Langevin recombination reduction factor $\gamma$. The currents have been normalized to the corresponding space-charge-limited current $J_{S C L C}=9 \varepsilon \varepsilon_{0}\left(\mu_{n}+\mu_{p}\right)\left(V-V_{b i}\right)^{2} /$ $8 d^{3}$, where $V$ is the applied voltage and $\varepsilon_{0}$ is the vacuum permittivity; the meaning of the other parameters are given in Table S2.

These films undergo a dramatic change in photovoltaic power conversion efficiency (PCE) as a result of subtle changes to film morphology—which is controlled by processing. As-cast 1:1 BQR:PC ${ }_{71} \mathrm{BM}$ bulk heterojunction devices (140nm thick) have PCEs of 4.2\%, increasing to 9.6\% after a brief THF solvent vapor annealing (SVA) treatment ${ }^{5}$ (Fig. 2a and Fig. S1). During the SVA process, the active layer swells with THF molecules, allowing BQR domains to undergo Ostwald ripening and form small but pure aggregates in the blend. This aggregation leads to the recovery of vibronic structure seen in Fig. 1b (green lines), and an increased (010) reflection in x-ray scattering data (see Fig. S2-S3), both indicating $\pi-\pi$ stacking. The quenching of BQR exciton emission with blend film formation (see Fig. S4) gives 93\% quenching for as-cast films compared to $79 \%$ for SVA, indicating that with annealing BQR domains increase in size and purity.

The drastic increase in device performance upon changes in morphology becomes particularly 
apparent for thicker junctions. For active layers with thickness of $320 \mathrm{~nm}$ (Fig. 2a), the as-cast device displays a relatively poor PCE of 3.45\% and Fill Factor (FF) of 41.4\%, whereas the SVA device shows a PCE of $9.75 \%$ and a FF of 74.3\% -exceptionally high for a $320 \mathrm{~nm}$ thick junction. A high PCE of $8.14 \%$ is maintained for $600 \mathrm{~nm}$ thick SVA devices (see Fig. S5) exhibiting an unprecedented FF at these thicknesses of 60.7\%. Resistance-dependent photo-voltage measurements on the as-cast device (Fig. 2b) reveal imbalanced mobilities of $1.5 \times 10^{-3} \mathrm{~cm}^{2} / \mathrm{Vs}$ and $4 \times 10^{-5} \mathrm{~cm}^{2} / \mathrm{Vs}$, assigned to electrons and holes, respectively. Furthermore, double injection (DoI) current transient measurements (Fig. 2c) on the as-cast device suggest a Langevin reduction factor of $\gamma \approx 10$. These findings are fortified by electro-optical device simulations (Fig. 2a) which reproduce the measured $J$ - $V$ characteristics, using the extracted transport parameters and complex refractive indices as input. Importantly, the simulations suggest that (electric-field dependent) geminate recombination is negligible under solar cell operating conditions.

The SVA device, in turn, exhibits fairly balanced mobilities (Fig. 2b) with a hole mobility of $8 \times$ $10^{-4} \mathrm{~cm}^{2} / \mathrm{Vs}$ and an electron mobility of $3 \times 10^{-3} \mathrm{~cm}^{2} / \mathrm{Vs}$. The increased hole mobility is consistent with larger and more pure BQR domains, expected upon SVA. Improved electron mobility (approaching that of neat $\mathrm{PC}_{71} \mathrm{BM}$ ), indicates better percolation between the $\mathrm{PC}_{71} \mathrm{BM}$ domains. DoI measurements on the SVA devices (Fig. 2c) reveal a heavily suppressed Langevin reduction factor of $\gamma=2000$. This reduction factor is the largest ever reported in a technologically relevant and efficient system. These results are corroborated by electro-optical device simulations (Fig. 2a), assuming negligible geminate recombination.

The drastically increased device performance upon SVA can thus be attributed to a significantly reduced recombination coefficient. While the measurements on as-cast devices can be explained by a recombination coefficient based on diffusion-controlled encounter of electrons and holes that are initially in separate phases, ${ }^{31}$ the impressive reduction of the recombination coefficient upon SVA cannot be explained based on charge carrier encounter in a simple two-component (donor:acceptor) system alone. Strong suppression of bimolecular recombination has been previously assigned to the fast dissociation of the charge transfer states relative to the decay rate of the singlet CT states as well as back electron transfer of the triplet CT states. ${ }^{32-34}$

To understand the fate of photogenerated charges, we probe their photoinduced absorption and also track their evolution using a striking and slowly-increasing Stark signature that is evident in 
transient data for the solvent vapor annealed films. In ultrafast transient absorption measurements, blend films were photo-excited at $600 \mathrm{~nm}$ in order to preferentially create excitons in the BQR donor. Figure 3a-d shows femtosecond transient spectra of $\mathrm{BQR}: \mathrm{PC} \mathrm{71}_{11} \mathrm{BM}$ blend films. As-cast films of $140 \mathrm{~nm}$ thickness (Fig. 3a) are compared to films having undergone SVA treatment; a 95 nm 'thin' film (Fig. 3b) and a $140 \mathrm{~nm}$ thicker film (Fig. 3c). The BQR exciton photoinduced absorption (PIA) signature was identified from measurements on neat BQR films (Fig. S6a and Fig. 3d), which can clearly be observed in all blend film transient spectra centered around 1100 nm, with a lifetime of 160 ps. A second PIA can also be observed in all three spectra from 700$950 \mathrm{~nm}$, corresponding to the BQR hole polaron as the result of charge transfer in the donoracceptor blend. This hole polaron band allows us to detect the generation of charges. Temporal slices of the BQR hole polaron region at $920 \mathrm{~nm}$ (Fig. 3e) reveal that charge transfer occurs predominately within the 200 fs instrument response time in all blend films despite differences in film crystallinity. As Samuel et al. have shown in their work on exciton diffusion, the increase in domain size is accompanied by an increase in exciton diffusion rate, therefore charge generation remains efficient even for large domains ${ }^{35}$. The as-cast film (Fig. 3a) also contains a further photoinduced absorption at 1100nm assigned to the BQR triplet species, which is discussed below.
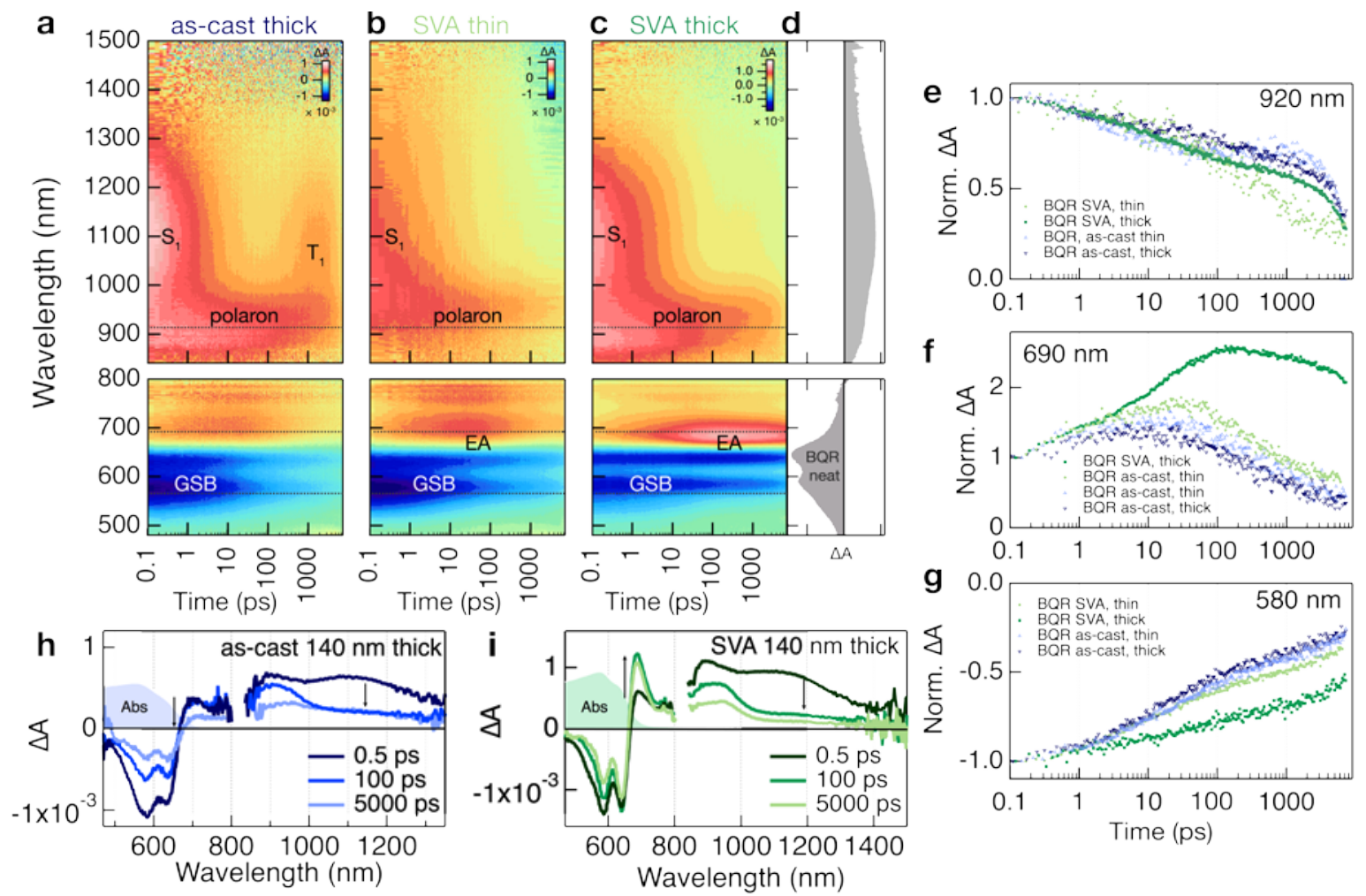
Figure 3. The results of ultrafast spectroscopy experiments (a) $140 \mathrm{~nm}$ thick BQR:PC ${ }_{71} \mathrm{BM}$ blend films ascast, (b) $95 \mathrm{~nm}$ thick BQR:PC ${ }_{71} \mathrm{BM}$ blend films after SVA and (c) $140 \mathrm{~nm}$ thick BQR:PC 71 BM blend films after SVA. (d) shows the transient spectra of the BQR exciton for comparison. Dominant species are labeled as 'GSB' (ground state bleach), 'EA' (electroabsorption); 'polaron' the BQR hole polaron, ' $\mathrm{S}_{1}$ ' the BQR exciton, and ' $\mathrm{T}_{1}$ ' the BQR triplet species. The normalized kinetics at different wavelengths are shown in (e) $920 \mathrm{~nm}$ (polaron PIA) (f) $690 \mathrm{~nm}$ (electro-absorption) and (g) $580 \mathrm{~nm}$ (ground state bleach) Spectral slices are shown for (h) the 'thick' 140nm as-cast blend film and (i) the 'thick'140nm SVA blend film.

In our transient absorption data, a strong electro-absorption signal (without any applied external field) is identified by its derivative-like signature around the BQR band-edge (620-720 nm) and is most pronounced in the $140 \mathrm{~nm}$ thick SVA sample (Fig. 3c). This Stark shift is caused by the field established due to charges that are intrinsically photogenerated in the active layer, rather than by any electrodes or external electric field. The chromophores affected by the electric field are overwhelmingly unexcited ground state molecules, due to the low excitation densities used and high probability that they will be in the vicinity of any fields formed. The normalized kinetics at $690 \mathrm{~nm}$ show a rise over $\sim 100$ ps accompanied by a preserved ground state bleach intensity (580 nm) (Fig. 3f-g). While the EA grow-in is striking in the thicker solvent vapor annealed film (Fig. 3i), the feature is limited in other samples (Fig. 3a, 3h and Figure S7), and is far less pronounced in thinner 95 nm SVA films; see Fig. $3 \mathbf{b}$.

For a Stark effect affecting a change of polarizability of a transition, interaction with an electric field will induce a dipole moment only in the direction of the field, regardless of the molecule's orientation, resulting in a first derivative lineshape of the EA signal ${ }^{13}$. In contrast, a change in dipole moment can result in either a first or second derivative signal depending on orientational effects. A well-defined relative orientation between a transition dipole moment of a chromphore, $\Delta \mu$, and an electric field would lead to a uni-directional shift of the ground state absorption and consequently a change in absorption with a first derivative lineshape (Fig. 4a) (see also supporting information section S4) ${ }^{20}$. 

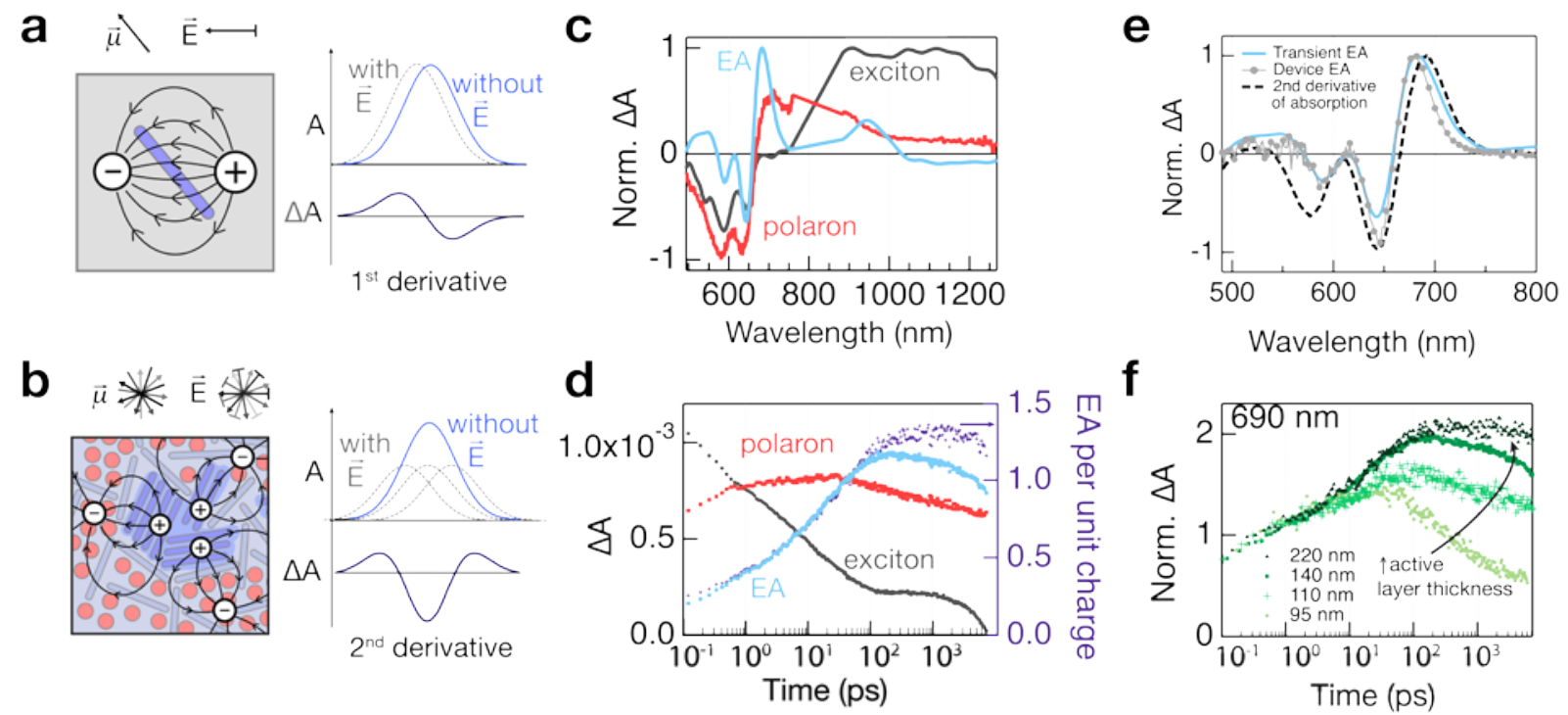

Figure 4. The electro-absorption signature. (a) The electro-absorption effect from an orientated chromophore where the transition dipole moment, $\Delta \vec{\mu}$, in an electric field, $\vec{E}$, results in a shift of the absorption spectrum, where the difference or Stark spectrum has a first derivative line shape. (b) When the electric field and chromophores are at a range of relative orientations, the difference spectrum appears as the second derivative of the ground-state absorption. (c) Extracted transient spectra from $140 \mathrm{~nm}$ SVA films, comprising an exciton (black), polaron (red) and EA signal (blue), with their dynamics shown in (d). (e) The extracted EA from the SVA blend film, steady-state device EA, and second derivative of the SVA $\mathrm{BQR}: \mathrm{PC}_{71} \mathrm{BM}$ ground state absorption spectrum. The extracted electro-absorption kinetic is divided by the polaron signal in (d) to give the magnitude of the EA contribution to the transient absorption per unit charge (right axis). (f) shows the increase in electroabsorption signal (at $690 \mathrm{~nm}$ ) for SVA BQR:PC ${ }_{71} B M$ active layers with identical processing but of increasing thickness.

Alternatively, a variety of orientations between many transition dipole moments, $\Delta \mu$, and electric field lines results in transition shifts to a range of both higher and lower energies-in other words the band broadens (Fig. 4a), resulting in a difference spectrum with a lineshape that is the second derivative of the ground state absorption ${ }^{12,13}$. When an external electric field is applied across a BQR:PC ${ }_{71} \mathrm{BM}$ bulk heterojunction device, a clear second derivative electro-absorption signal from the BQR component is observed in the steady-state absorption with increasing applied voltage (Fig S10a and device EA in Fig. 4e).

By fitting this steady-state EA, BQR has a transition dipole moment, $\Delta \vec{\mu}$, of 8.43 Debye, typical for a Frenkel exciton, and small change in polarizability, $\Delta \alpha$, of $35.38 \AA^{3}$. This indicates that the BQR transition dipole rather than the polarizability change is predominantly responsible for the Stark response of the material, hence the dominant second derivative spectral shape (see Fig. S10 
and S11). The ground state dipole is not expected to have a significant impact in this system; from DFT calculations (see Figure S12) BQR has a very small ground state dipole moment, $\vec{\mu}$, of 1.16 $\times 10^{-4}$ Debye, and a ground state polarizability, $\alpha$, of $315.33 \AA^{3}$.

To monitor the EA signature directly, the 3D transient surface was decomposed into its principal spectra and kinetics (Fig. 4c-d) via global analysis based on a genetic algorithm ${ }^{20}$, where the neat BQR exciton and polaron spectra were used in the fitting procedure (see supporting information, section S5 and Fig. S13). The data can be well-described by three components, the extracted kinetics showing that excitons form hole polarons either within the instrument response time or soon after. The electro-absorption component resembles the second derivative of the ground state SVA BQR:PC $71 B M$ absorption spectrum, and very closely resembles the steady-state electroabsorption of BQR excitons in a device with applied macroscopic electric field (Fig. 4e and Fig. S10). The EA signal intensity increases from almost zero over 100s of picoseconds, while concurrently the polaron signal is maintained.

The signal is caused by electrons and holes generated within the film, where the electric fields between these carriers perturb the ground state optical absorption of surrounding chromophores. Interestingly, and in contrast to the dynamics of other species in the transient absorption data, the EA dynamics are significantly dependent on device thickness (Fig. 4f and Fig. S14), which is discussed further below. The EA dynamics are also very sensitive to pump power (Fig. S9a), down to $\sim 5 \mu \mathrm{J} / \mathrm{cm}^{2}$, which along with their pico- to nanosecond dynamics indicates that they are caused by free charge carriers. A second derivative lineshape also infers that the signal corresponds to a population of many dipole orientations relative to that of the electric fields ${ }^{12}$ (Fig. $4 \mathbf{b}$ ). The EA signal shown here contrasts with previous reports in the literature in that here the migration of free carriers is responsible for this Stark effect, rather than fast initial charge separation. Previous reports show intrinsic electro-absorption effects in organic bulk heterojunctions on much shorter 25 timescales, decaying immediately after photoexcitation due to the initial charge separation events at an interface ${ }^{19,21,22,36,37}$. In a variety of systems, these signals manifest as first derivative Stark signals, and persist and then decay on the timescales of charge separation, generally $100 \mathrm{fs}$ or, in rare cases, a few picoseconds ${ }^{21}$. The conventional wisdom on this topic describes an electric field between charges decaying as carriers diffuse away from one another ${ }^{38}$. However, we see the opposite, with a field increasing in magnitude up to 1 nanosecond as a capacitive build-up occurs. After this time, the magnitude of the EA signal is maintained on the timescale of $>1$ ns, though 
longer time transient data reveal that it does not remain on the timescale $>100$ ns (Fig. S8), suggesting that it would not act as a terminal trap for charge carriers.

An interesting control is provided by $\mathrm{BQR}: \mathrm{PC}_{71} \mathrm{BM}$ active layers that are not solvent vapor annealed-we do not observe any electric field buildup in as-cast BQR:PC ${ }_{71} B M$ active layers. Rather, polarons are lost via recombination of free charges to BQR triplet excitons ${ }^{33,39-41}$. The formation of triplets occurs on the same timescale as the polaron decay and can be seen as a photoinduced absorption in Figure 3a, centered at $1050 \mathrm{~nm}$ and appearing 200 ps after excitation (further details are provided in Fig. S15). The formation of this species is analogous to the recombination of injected charges in an LED, where spin-uncorrelated free charges recombine at a 3:1 ratio of triplet:singlet charge transfer states (Fig. 5a-b).

We do not see any evidence that geminate recombination contributes as a significant decay pathway in the as-cast films. Firstly, the polaron signal and triplet signal of as-cast films are powerdependent, (Figure S9b and S15b respectively) indicating that bimolecular recombination dominates here. Secondly, by comparing the ground state bleaches (Figure 3g), the 90nm (thinner) as-cast and SVA samples have very similar bleach recovery dynamics. The device performance is very different, but with similar bleaches, geminate recombination to the ground state does not appear to be a major contributor. When comparing the thicker $140 \mathrm{~nm}$ films, the SVA film has a significant EA contribution to its bleach, (Fig. 3c) making it more difficult to compare this dynamic. Finally, $J-V$ simulations (Fig. 2a) give an excellent match to experiment and suggest that 20 (electric-field dependent) geminate recombination is negligible under solar cell operating conditions. 

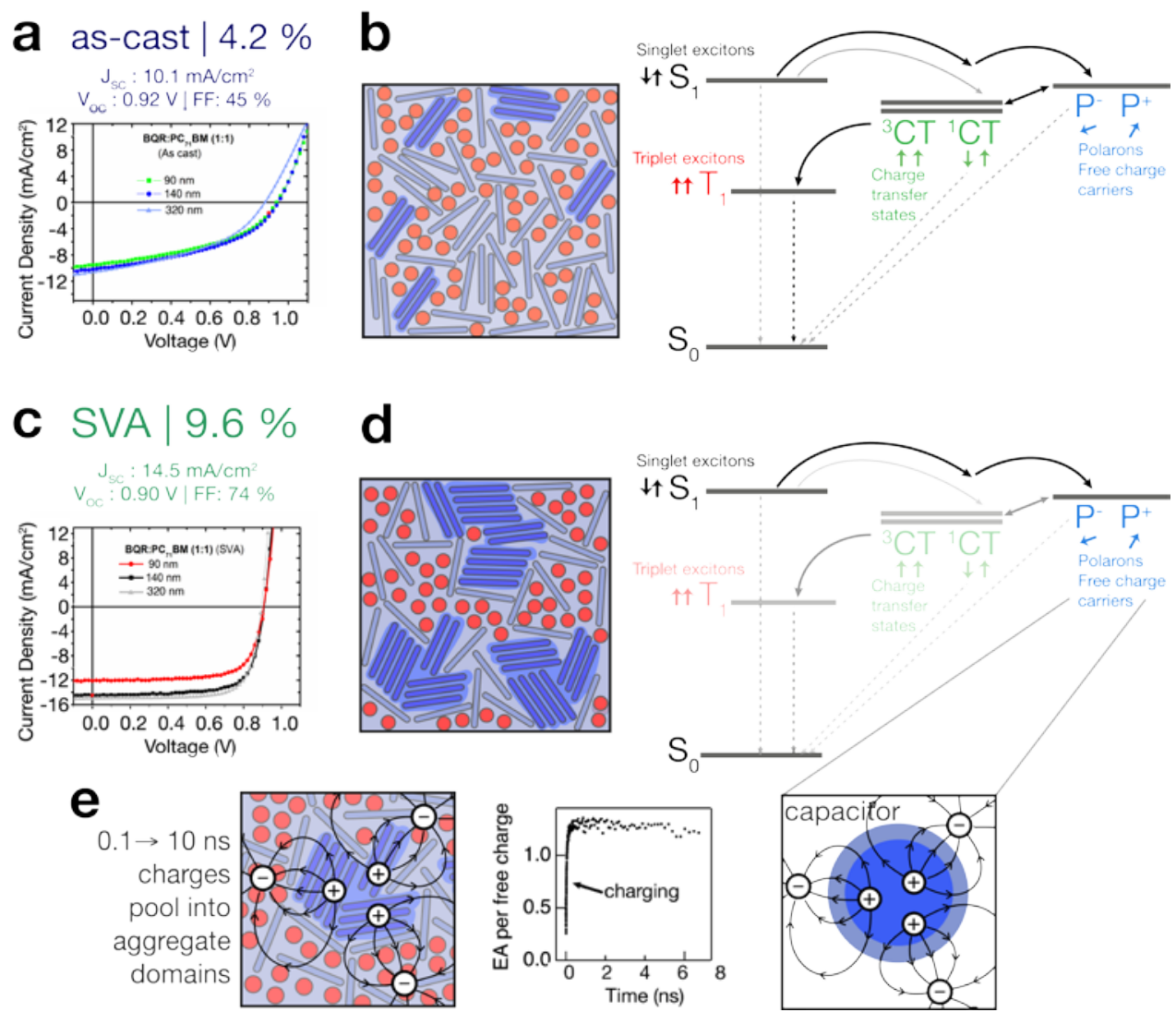

Figure 5. The fate of charge carriers (a) $J$ - $V$ curves and efficiencies for as-cast BQR:PC ${ }_{71} B M$ blend (140 nm thick) where (b) shows a schematic of the morphology and dominant charge generation pathways. (c) $J$ - $V$ curves and efficiencies for solvent vapor annealed BQR:PC ${ }_{71} \mathrm{BM}$ blend (140 nm thick) where (d) shows a schematic of the morphology and the dominant charge generation pathway, where a strong electroabsorption build-up is present. (e) shows the aggregate domain charge pooling that causes the EA signature to build and then be maintained over nanosecond timescales and its resemblance to charge buildup in a capacitor.

The distinctive pathways for free charges shown here are reflected in devices made from these active layers. As-cast devices (140nm thick), with the bimolecular loss pathway to the triplet exciton, have efficiencies of $\sim 4.2 \%$ (Fig. $5 \mathbf{a}$ and $\mathbf{b}$ ) with an open-circuit voltage of $0.92 \mathrm{~V}$ and fill factor of $45 \%$. Following SVA, device efficiencies increase to $9.6 \%$, largely retaining an opencircuit voltage of $0.90 \mathrm{~V}$ yet with a significantly improved fill factor of $72 \%$, owing to the reduced bimolecular recombination (Fig. 5c and d).

The EA kinetic trace normalized by the charge population gives the amount of EA per unit charge in the active layer (Fig. 4d, right axis) where the EA signal increases for 100s of picoseconds and saturates at the nanosecond timescale. We note that this timescale is at least one order of magnitude 
faster than the normally reported fastest carrier transit times, indicating that this effect would not necessarily result in poor carrier diffusion. The EA signal rise is also correlated with a red-shift of the polaron signal maximum over time (Fig. 3c). This indicates that the electric field builds as hole polarons migrate from intermixed higher energy regions and pool in lower energy BQR aggregates. This energetic cascade can provide a driving force to enable spatial separation of electrons and holes, and has been shown to lead to longer-lived populations of extractable charges ${ }^{42,43}$. Further evidence to support the importance of a morphological energetic cascade can be seen by measuring the transient spectra of a blend with a dilute acceptor component. We see the complete absence of the electro-absorption signal when acceptor molecules are isolated in the blend, by examining a 95:5 BQR:PC ${ }_{71}$ BM SVA film (Fig. S16). Excitons undergo charge separation to form polarons within this blend, however, we see no evidence of charge buildup or capacitive charging due to the absence of the cascade needed to form such a charge buildup.

The electric field buildup may be also caused by built-in nanoscale electrostatic effects within the active layer, fortifying the energetic cascade. The collective action of the anisotropic distribution of electronic density in typical aggregated conjugated molecules has been discussed widely in the theory and simulation of organic aggregates ${ }^{44-47}$, though is difficult to measure experimentally, especially at buried interfaces. This effect has been calculated to significantly alter the energetic landscape for charge carriers in aggregate films ${ }^{46}$. While static microscopic electric fields caused only by molecular arrangement can cause built-in electric fields, this effect would cancel in a differential transient absorption measurement. However, a flow of migrating photoinduced charge carriers as directed by the potential energy landscape of built-in fields would be evident in an electro-absorption signal in such a pump-on, pump-off experiment.

In contrast to photoinduced absorption signals, which should scale linearly with film thickness, the electro-absorption signature exhibits a more significant thickness-dependent behavior in SVA films (Fig. 4f, Fig. 3e-g and Fig. S14). Charge carriers produce local electric fields, where the amount of $\Delta A$ signal originating from the Stark effect is proportional to the electric field squared, $\vec{E}^{2}$. Thicker films contain a greater number of photogenerated carriers, leading to a more significant scaling in the EA signal than a standard optical transition with increasing active layer thickness. We note that this phenomenon was only easily detectable in transient absorption measurements with relatively thick junctions. Consequently, it is possible that there are existing systems where this same effect may be present, but could have remained undetected. 
The charge migration and large electric field buildup observed here in the SVA heterojunction is analogous to the charging of a capacitor (Fig. 5e). We emphasize that all transient experiments were undertaken on only the device active layer, equivalent to a device at $\mathrm{V}_{\mathrm{OC}}$. The addition of electrodes in a full device would provide an additional electric field aiding in the extraction of charges. Charges first stored away from the interface over nanosecond timescales can later be harvested at electrodes through drift and diffusion over the expected micro-millisecond timescales for carrier migration. We note that this result contrasts with the accepted idea of an ideal open circuit device where radiative recombination should be at a maximum ${ }^{48}$. It shows that morphology can influence the fate of charges across interfaces for the first nanoseconds following photoexcitation, potentially suggesting a more nuanced way to understand charge migration and $V_{O C}$ in organic photovoltaics. The presence of interdomain electric fields could allow for the existence of charge throughout the device, which would prevent the presence of space charge regions at contacts that limit fill factors ${ }^{49}$. The devices with SVA treatment are able to maintain good efficiencies in even thicker active layers than those studied here, up to $8.1 \%$ in $600 \mathrm{~nm}$ active layer and a fill factor of $61 \%$ (Table S1). The relationship between efficient thick heterojunctions and the capacitive charging reported here is the topic of ongoing and future investigations.

Steady-state electro-absorption measurements (Fig. S10a and b) were used to calibrate the observed Stark effect. The observed field at its maximum value is estimated at $487 \mathrm{kV} / \mathrm{cm}$, extremely large compared to that expected locally between domains, and unprecedented in photovoltaic systems. This value is somewhat comparable to fields generated from charges that are held in nanoscale proximity such as in ultracapacitors ${ }^{50}$ and photogenerated ion pairs ${ }^{51}$. The relative dielectric constants of organic semiconductors are generally between 2 and 4, which is comparable to that of a dielectric layer in a standard macroscopic capacitor. Due to this dielectric environment, the electric fields responsible for the EA will polarize the intervening medium between regions of high charge density — causing charges to be further shielded from their mutual attraction at the interface. This suggests that in devices where charge separation can occur efficiently, the low dielectric constant (often cited as a hindrance in organic photovoltaics) could actually help carriers avoid bimolecular recombination. 
Our results show that free charges can build up in crystalline domains in an organic bulk heterojunction film, with large electric fields of up to $\sim 487 \mathrm{kV} / \mathrm{cm}$ generated between positive and negative carriers. Rather than a decay of the electric field as charges separate over time, we see the opposite, with a field increasing in magnitude up to 1 nanosecond as a capacitive build-up occurs. Charges must be in close proximity to one another $(<5 \mathrm{~nm})$ in order to form and maintain such large electric fields. Surprisingly, both spectroscopic, device and charge recombination data show that charges are protected by this capacitive charging and recombination is substantially suppressed by up to 2000 times compared to the Langevin rate.

We show that charges are protected over the early timescales of carrier migration and that the resulting capacitive charging is not detrimental to the migration of charges as it is for traps and defects. These observations are particularly interesting given the debate regarding how photogenerated charge-pairs escape and avoid the predicted strong Coulombic attraction between carriers in organic heterojunctions and how only some materials can support thick junctions. $7,52,53$ Given that the transient signature of these electric fields may be easily obscured in spectroscopic measurements-only appearing clearly in thicker active layers-we speculate that this phenomenon might be found in other material blends, especially those that support efficient thick devices and have suppressed recombination. Beyond simply the role of donor and acceptor components, we show that the local environment of interfaces and their energetic landscapes can make a key contribution to the fate of photogenerated charges within a photovoltaic system. The

20 capacitor-like charge buildup we observe shows that nanomorphology and electric fields in photovoltaics can play an unexpected role in protecting photogenerated charges from recombination, thus suggesting a significant new design principle for optimizing OPV devices.

\section{Acknowledgments:}

K.N.S would like to acknowledge Matthew Menke and Lee Richter for helpful discussions, and Simone Gelinas for contibutions to developing the genetic algorithm software. Funding: This work was made possible by support from the Australian Renewable Energy Agency, which funds the project grants within the Australian Centre for Advanced Photovoltaics. K.N.S acknowledges the Australian Renewable Energy Agency for a postgraduate scholarship. G.D.S and K.N.S. acknowledge support by the U.S. Department of Energy, Office of Science, Office of Basic 30 Energy Sciences Solar Photochemistry program under Award Number DE-SC0015429. S.U.Z.K. 
and B.P.R. acknowledge funding from the U.S. Department of Energy, Office of Basic Energy Sciences, Division of Materials Sciences and Engineering under Award Number DESC0012458. A.A. is a Sêr Cymru II Rising Star Fellow supported by European Regional Development Fund, Welsh European Funding Office and Swansea University strategic initiative in Sustainable Advanced Materials. B.K. acknowledges support by the National Science Foundation Graduate Research Fellowship under Grant Number DGE-1656466. Competing interests: Authors declare no competing interests. Data and materials availability: All data is available in the main text or the supporting information.

\section{Supporting Information:}

Figures S1-S16

References

\section{References and Notes:}

(1) Polman, A.; Knight, M.; Garnett, E. C.; Ehrler, B.; Sinke, W. C. Photovoltaic Materials: Present Efficiencies and Future Challenges. Science 2016, 352 (6283), aad4424. https://doi.org/https://doi.org/10.1126.

(2) Brédas, J.-L.; Norton, J. E.; Cornil, J.; Coropceanu, V. Molecular Understanding of Organic Solar Cells: The Challenges. Acc. Chem. Res. 2009, 42 (11), 1691-1699. https://doi.org/10.1021/ar900099h.

(3) Blankenship, R. E. Molecular Mechanisms of Photosynthesis; Blackwell Science, 2002.

(4) Scherf, U.; Harth, E.; Gügel, A.; Müllen, K.; Lemmer, U.; Feldmann, J. Exciton Diffusion and Dissociation in Conjugated Polymer/Fullerene Blends and Heterostructures. Phys. Rev. B - Condens. Matter Mater. Phys. 1999, 59 (23), 15346-15351. https://doi.org/10.1103/PhysRevB.59.15346.

(5) Collins, S. D.; Ran, N. A.; Heiber, M. C.; Nguyen, T.-Q. Small Is Powerful: Recent Progress in Solution-Processed Small Molecule Solar Cells. Adv. Energy Mater. 2017, 7 (10), 1602242.

(6) Lakhwani, G.; Rao, A.; Friend, R. H. Bimolecular Recombination in Organic 
Photovoltaics. Annu. Rev. Phys. Chem. 2014, 65 (1), 557-581.

https://doi.org/10.1146/annurev-physchem-040513-103615.

(7) Meredith, P.; Armin, A. Scaling of next Generation Solution Processed Organic and Perovskite Solar Cells. Nat. Commun. 2018, 9 (1), 8-11. https://doi.org/10.1038/s41467018-05514-9.

(8) Armin, A.; Chen, Z.; Jin, Y.; Zhang, K.; Huang, F.; Shoaee, S. A Shockley-Type Polymer: Fullerene Solar Cell. Adv. Energy Mater. 2018, 8 (7), 1-9.

https://doi.org/10.1002/aenm.201701450.

(9) Armin, A.; Subbiah, J.; Stolterfoht, M.; Shoaee, S.; Xiao, Z.; Lu, S.; Jones, D. J.;

Meredith, P. Reduced Recombination in High Efficiency Molecular Nematic Liquid Crystalline: Fullerene Solar Cells. Adv. Energy Mater. 2016, 6 (22), 1-10. https://doi.org/10.1002/aenm.201600939.

(10) Jin, Y.; Chen, Z.; Xiao, M.; Peng, J.; Fan, B.; Ying, L.; Zhang, G.; Jiang, X.-F.; Yin, Q.; Liang, Z.; Huang, F.; Cao, Y. Thick Film Polymer Solar Cells Based on Naphtho[1,2c :5,6- c ]Bis[1,2,5]Thiadiazole Conjugated Polymers with Efficiency over 11\%. Adv. Energy Mater. 2017, 7 (22), 1700944. https://doi.org/10.1002/aenm.201700944.

(11) Langevin, M. Recombinaison et Diffusion Des Ions Gazeux. J. Phys. Theor. Appl. 1905, 4 (1), 322-333.

(12) Bublitz, G.; Boxer, S. Stark Spectroscopy: Applications in Chemistry, Biology, and Materials Science. Annu. Rev. Phys. Chem. 1997, 48, 213-242. https://doi.org/10.1146/annurev.physchem.48.1.213.

(13) Boxer, S. Stark Realities. J. Phys. Chem. B 2009, 112 (41), 592-602.

(14) Gulbinas, V.; Kananavičius, R.; Valkunas, L.; Bässler, H. Dynamic Stark Effect as a Probe of the Evolution of Geminate Electron-Hole Pairs in a Conjugated Polymer. Phys. Rev. B - Condens. Matter Mater. Phys. 2002, 66 (23), 1-4. https://doi.org/10.1103/PhysRevB.66.233203.

(15) Cabanillas-Gonzalez, J.; Virgili, T.; Gambetta, A.; Lanzani, G.; Anthopoulos, T. D.; De Leeuw, D. M. Photoinduced Transient Stark Spectroscopy in Organic Semiconductors: A Method for Charge Mobility Determination in the Picosecond Regime. Phys. Rev. Lett. 
2006, 96 (10), 6-9. https://doi.org/10.1103/PhysRevLett.96.106601.

(16) Klimov, V. I. Spectral and Dynamical Properties of Multiexcitons in Semiconductor Nanocrystals. Annu. Rev. Phys. Chem. 2007, 58, 635-673.

https://doi.org/10.1146/annurev.physchem.58.032806.104537.

(17) Ardo, S.; Sun, Y.; Castellano, F. N.; Meyer, G. J. Excited-State Electron Transfer from Ruthenium-Polypyridyl Compounds to Anatase TiO2 Nanocrystallites: Evidence for a Stark Effect. J. Phys. Chem. B 2010, 114 (45).

(18) Koch, M.; Myahkostupov, M.; Oblinsky, D. G.; Wang, S.; Garakyaraghi, S.; Castellano, F. N.; Scholes, G. D. Charge Localization after Ultrafast Photoexcitation of a Rigid Perylene Perylenediimide Dyad Visualized by Transient Stark Effect. J. Am. Chem. Soc. 2017, 139 (15), 5530-5537. https://doi.org/10.1021/jacs.7b01630.

(19) Scarongella, M.; De Jonghe-Risse, J.; Buchaca-Domingo, E.; Causa, M.; Fei, Z.; Heeney, M.; Moser, J. E.; Stingelin, N.; Banerji, N. A Close Look at Charge Generation in Polymer: Fullerene Blends with Microstructure Control. J. Am. Chem. Soc. 2015, 137 (8), 2908-2918. https://doi.org/10.1021/ja510032x.

(20) Gelinas, S.; Rao, A.; Kumar, A.; Smith, S. L.; Chin, A. W.; Clark, J.; van der Poll, T. S.; Bazan, G. C.; Friend, R. H. Ultrafast Long-Range Charge Separation in Organic Semiconductor Photovoltaic Diodes. Science 2014, 343 (6170), 512-516. https://doi.org/10.1126/science.1246249.

(21) Menke, S. M.; Cheminal, A.; Conaghan, P.; Ran, N. A.; Greehnam, N. C.; Bazan, G. C.; Nguyen, T. Q.; Rao, A.; Friend, R. H. Order Enables Efficient Electron-Hole Separation at an Organic Heterojunction with a Small Energy Loss. Nat. Commun. 2018, 9 (1), 1-7. https://doi.org/10.1038/s41467-017-02457-5.

(22) Tamai, Y.; Fan, Y.; Kim, V. O.; Ziabrev, K.; Rao, A.; Barlow, S.; Marder, S. R.; Friend, R. H.; Menke, S. M. Ultrafast Long-Range Charge Separation in Nonfullerene Organic Solar Cells. ACS Nano 2017, 11 (12), 12473-12481. https://doi.org/10.1021/acsnano.7b06575.

(23) Geraghty, P. B.; Lee, C.; Subbiah, J.; Wong, W. W. H.; Banal, J. L.; Jameel, M. A.; Smith, T. A.; Jones, D. J. High Performance P-Type Molecular Electron Donors for OPV 
Applications via Alkylthiophene Catenation Chromophore Extension. Beilstein J. Org. Chem. 2016, 12 (Mm), 2298-2314. https://doi.org/10.3762/bjoc.12.223.

(24) Sandberg, O. J.; Nyman, M.; Österbacka, R. Effect of Contacts in Organic Bulk Heterojunction Solar Cells. Phys. Rev. Appl. 2014, 1 (2), 1-15. https://doi.org/10.1103/PhysRevApplied.1.024003.

(25) Pettersson, L. A. A.; Roman, L. S.; Inganäs, O. Modeling Photocurrent Action Spectra of Photovoltaic Devices Based on Organic Thin Films. J. Appl. Phys. 1999, 86 (1), 487-496. https://doi.org/10.1063/1.370757.

(26) Sugunan, S. K.; Robotham, B.; Sloan, R. P.; Szmytkowski, J.; Ghiggino, K. P.; Paige, M. F.; Steer, R. P. Photophysics of Untethered ZnTPP-Fullerene Complexes in Solution. J. Phys. Chem. A 2011, 115 (44), 12217-12227. https://doi.org/10.1021/jp2082853.

(27) Stevens, M. A.; Silva, C.; Russell, D. M.; Friend, R. H. Exciton Dissociation Mechanisms in the Polymeric Semiconductors Poly(9,9-Dioctylfluorene) and Poly(9,9-DioctylfluoreneCo-Benzothiadiazole). Phys. Rev. B 2001, 63 (16), 165213. https://doi.org/10.1103/PhysRevB.63.165213.

(28) Ferguson, A. J.; Kopidakis, N.; Shaheen, S. E.; Rumbles, G. Quenching of Excitons by Holes in Poly(3-Hexylthiophene) Films. J. Phys. Chem. C 2008, 112 (26), 9865-9871. https://doi.org/10.1021/jp7113412.

(29) Hodgkiss, J. M.; Albert-Seifried, S.; Rao, A.; Barker, A. J.; Campbell, A. R.; Marsh, R. A.; Friend, R. H. Exciton-Charge Annihilation in Organic Semiconductor Films. Adv. Funct. Mater. 2012, 22 (8), 1567-1577. https://doi.org/10.1002/adfm.201102433.

(30) Frisch, M. J.; Trucks, G. W.; Schlegel, H. E.; Scuseria, G. E.; Robb, M. A.; Cheeseman, J. R.; Scalmani, G.; Barone, V.; Petersson, G. A.; Farkas, O.; Foresman, J. B.; Fox, J. D. Gaussian 16. Gaussian, Inc., Wallingford CT,. 2016.

(31) Heiber, M. C.; Nguyen, T. Q.; Deibel, C. Charge Carrier Concentration Dependence of Encounter-Limited Bimolecular Recombination in Phase-Separated Organic Semiconductor Blends. Phys. Rev. B 2016, 93 (20), 1-8. https://doi.org/10.1103/PhysRevB.93.205204.

(32) Shoaee, S.; Armin, A.; Stolterfoht, M.; Hosseini, S. M.; Kurpiers, J.; Neher, D. Decoding 
Charge Recombination through Charge Generation in Organic Solar Cells. Sol. RRL 2019, 1900184, 1900184. https://doi.org/10.1002/solr.201900184.

(33) Schwarz, K. N.; Geraghty, P. B.; Jones, D. J.; Smith, T. A.; Ghiggino, K. P. Suppressing Subnanosecond Bimolecular Charge Recombination in a High-Performance Organic

Photovoltaic Material. J. Phys. Chem. C 2016, 120 (42), 24002-24010.

https://doi.org/10.1021/acs.jpcc.6b08354.

(34) Rao, A.; Chow, P. C. Y.; Gélinas, S.; Schlenker, C. W.; Li, C.-Z.; Yip, H.-L.; Jen, A. K.Y.; Ginger, D. S.; Friend, R. H. The Role of Spin in the Kinetic Control of Recombination in Organic Photovoltaics. Nature 2013, 500 (7463), 435-439. https://doi.org/10.1038/nature12339.

(35) Sajjad, M. T.; Zhang, Y.; Geraghty, P. B.; Mitchell, V. D.; Ruseckas, A.; Blaszczyk, O.; Jones, D. J.; Samuel, I. D. W. Tailoring Exciton Diffusion and Domain Size in Photovoltaic Small Molecules by Annealing. J. Mater. Chem. C 2019, 7 (26), 7922-7928. https://doi.org/10.1039/C9TC00951E.

(36) Gelinas, S.; Rao, A.; Kumar, A.; Smith, S. L.; Chin, A. W.; Clark, J.; van der Poll, T. S.; Bazan, G. C.; Friend, R. H. Ultrafast Long-Range Charge Separation in Organic Semiconductor Photovoltaic Diodes. Science 2014, 343 (6170), 512-516. https://doi.org/10.1126/science.1246249.

(37) Jakowetz, A. C.; Böhm, M. L.; Sadhanala, A.; Huettner, S.; Rao, A.; Friend, R. H. Visualizing Excitations at Buried Heterojunctions in Organic Semiconductor Blends. Nat. Mater. 2017, 16 (5), 551-557. https://doi.org/10.1038/nmat4865.

(38) Vithanage, D. A.; Devižis, A.; Abramavičius, V.; Infahsaeng, Y.; Abramavičius, D.; MacKenzie, R. C. I.; Keivanidis, P. E.; Yartsev, A.; Hertel, D.; Nelson, J.; Sundström, V.; Gulbinas, V. Visualizing Charge Separation in Bulk Heterojunction Organic Solar Cells. Nat. Commun. 2013, 4 (1), 2334. https://doi.org/10.1038/ncomms3334.

(39) Rao, A.; Chow, P. C. Y.; Gélinas, S.; Schlenker, C. W.; Li, C. Z.; Yip, H. L.; Jen, A. K. Y.; Ginger, D. S.; Friend, R. H. The Role of Spin in the Kinetic Control of Recombination in Organic Photovoltaics. Nature 2013, 500 (7463), 435-439. https://doi.org/10.1038/nature12339. 
(40) Gehrig, D. W.; Howard, I. A.; Laquai, F. F. Charge Carrier Generation Followed by Triplet State Formation , Annihilation , and Carrier Recreation in PBDTTT-C / PC 60 BM Photovoltaic Blends. J. Phys. Chem. C 2015, 119 (24), 13509-13515. https://doi.org/10.1021/acs.jpcc.5b03467.

(41) Jackson, N. E.; Savoie, B. M.; Marks, T. J.; Chen, L. X.; Ratner, M. A. The Next Breakthrough for Organic Photovoltaics? J. Phys. Chem. Lett. 2015, 6 (1), 77-84. https://doi.org/10.1021/jz502223t.

(42) Vandewal, K.; Himmelberger, S.; Salleo, A. Structural Factors That Affect the Performance of Organic Bulk Heterojunction Solar Cells. Macromolecules 2013, 46 (16), 6379-6387. https://doi.org/10.1021/ma400924b.

(43) Gallaher, J. K.; Prasad, S. K. K.; Uddin, M. A.; Kim, T.; Kim, J. Y.; Woo, H. Y.; Hodgkiss, J. M. Spectroscopically Tracking Charge Separation in Polymer : Fullerene Blends with a Three-Phase Morphology. Energy Environ. Sci. 2015, 8 (9), 2713-2724. https://doi.org/10.1039/C5EE01713K.

(44) Heimel, G.; Salzmann, I.; Duhm, S.; Koch, N. Design of Organic Semiconductors from Molecular Electrostatics. Chem. Mater. 2011, 23 (3), 359-377. https://doi.org/10.1021/cm1021257.

(45) D’Avino, G.; Muccioli, L.; Castet, F.; Poelking, C.; Andrienko, D.; Soos, Z. G.; Cornil, J.; Beljonne, D. Electrostatic Phenomena in Organic Semiconductors: Fundamentals and Implications for Photovoltaics. J. Phys. Condens. Matter 2016, 28 (43), 433002. https://doi.org/10.1088/0953-8984/28/43/433002.

(46) Cornil, J.; Verlaak, S.; Martinelli, N.; Mityashin, A.; Olivier, Y.; Van Regemorter, T.; D’Avino, G.; Muccioli, L.; Zannoni, C.; Castet, F.; Beljonne, D.; Heremans, P. Exploring the Energy Landscape of the Charge Transport Levels in Organic Semiconductors at the Molecular Scale. Acc. Chem. Res. 2013, 46 (2), 434-443. https://doi.org/10.1021/ar300198p.

(47) Tsiper, E. V.; Soos, Z. G. Charge Redistribution and Polarization Energy of Organic Molecular Crystals. Phys. Rev. B - Condens. Matter Mater. Phys. 2001, 64 (19), 1-12. https://doi.org/10.1103/PhysRevB.64.195124. 
(48) Miller, O. D.; Yablonovitch, E.; Kurtz, S. R. Strong Internal and External Luminescence as Solar Cells Approach the Shockley-Queisser Limit. IEEE J. Photovoltaics 2012, 2 (3), 303-311. https://doi.org/10.1109/JPHOTOV.2012.2198434.

(49) Tait, J. G.; Paetzold, U. W.; Cheyns, D.; Turbiez, M.; Heremans, P.; Rand, B. P. Interfacial Depletion Regions: Beyond the Space Charge Limit in Thick Bulk Heterojunctions. ACS Appl. Mater. Interfaces 2016, 8 (3), 2211-2219. https://doi.org/10.1021/acsami.5b10891.

(50) Stoller, M. D.; Park, S.; Zhu, Y.; An, J.; Ruoff, R. S. Graphene-Based Ultracapacitors. Nano Lett. 2008, 8 (10), 3498-3502. https://doi.org/10.1021/nl802558y.

(51) Gosztola, D.; Yamada, H.; Wasielewski, M. R. Electric Field Effects of Photogenerated Ion Pairs on Nearby Molecules: A Model for the Carotenoid Band Shift in Photosynthesis. J. Am. Chem. Soc. 1995, 117 (7), 2041-2048. https://doi.org/10.1021/ja00112a018.

(52) Bakulin, A.; Rao, A.; Pavelyev, V. G.; van Loosdrecht, P. H. M.; Pshenichnikov, M. S.; Niedzialek, D.; Cornil, J.; Beljonne, D.; Friend, R. H. The Role of Driving Energy and Delocalized States for Charge Separation in Organic Semiconductors. Science 2012, 335 (6074), 1340-1344. https://doi.org/10.1126/science.1217745.

(53) Vandewal, K.; Albrecht, S.; Hoke, E. T.; Graham, K. R.; Widmer, J.; Douglas, J. D.; Schubert, M.; Mateker, W. R.; Bloking, J. T.; Burkhard, G. F.; Sellinger, A.; Fréchet, J. M. J.; Amassian, A.; Riede, M. K.; McGehee, M. D.; Neher, D.; Salleo, A. Efficient Charge Generation by Relaxed Charge-Transfer States at Organic Interfaces. Nat. Mater. 2014, 13 (1), 63-68. https://doi.org/10.1038/nmat3807.

\section{TOC image:}

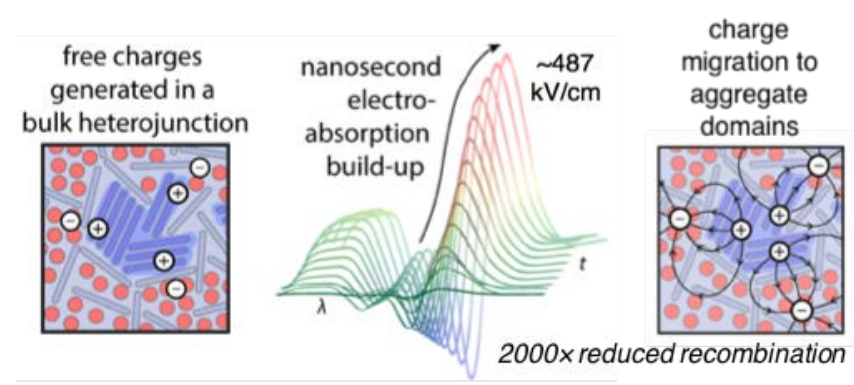

